\title{
Scenario-Mining for Level 4 Automated Vehicle Safety Assessment from Real Accident Situations in Urban Areas Using a Natural Language Process
}

\author{
Sangmin Park (D), Sungho Park, Harim Jeong, Ilsoo Yun (D) and Jaehyun (Jason) So *(D) \\ Department of Transportation Engineering, Ajou University, Suwon 16499, Korea; stylecap@ajou.ac.kr (S.P.); \\ fenix3339@ajou.ac.kr (S.P.); gkfla0731@ajou.ac.kr (H.J.); ilsooyun@ajou.ac.kr (I.Y.) \\ * Correspondence: jso@ajou.ac.kr
}

Citation: Park, S.; Park, S.; Jeong, H.; Yun, I.; So, J. Scenario-Mining for Level 4 Automated Vehicle Safety Assessment from Real Accident Situations in Urban Areas Using a Natural Language Process. Sensors 2021, 21, 6929. https://doi.org/ $10.3390 / \mathrm{s} 21206929$

Academic Editor: Felipe Jiménez

Received: 12 September 2021

Accepted: 14 October 2021

Published: 19 October 2021

Publisher's Note: MDPI stays neutral with regard to jurisdictional claims in published maps and institutional affiliations.

Copyright: (c) 2021 by the authors. Licensee MDPI, Basel, Switzerland. This article is an open access article distributed under the terms and conditions of the Creative Commons Attribution (CC BY) license (https:// creativecommons.org/licenses/by/ $4.0 /)$.

\begin{abstract}
As the research and development activities of automated vehicles have been active in recent years, developing test scenarios and methods has become necessary to evaluate and ensure their safety. Based on the current context, this study developed an automated vehicle test scenario derivation methodology using traffic accident data and a natural language processing technique. The natural language processing technique-based test scenario mining methodology generated 16 functional test scenarios for urban arterials and 38 scenarios for intersections in urban areas. The proposed methodology was validated by determining the number of traffic accident records that can be explained by the resulting test scenarios. That is, the resulting test scenarios are valid and represent a matching rate between the test scenarios and the increased number of traffic accident records. The resulting functional scenarios generated by the proposed methodology account for $43.69 \%$ and $27.63 \%$ of the actual traffic accidents for urban arterial and intersection scenarios, respectively.
\end{abstract}

Keywords: automated vehicle; scenario-mining; safety; natural language process; accident data

\section{Introduction}

The test scenario is a key measure for evaluating and ensuring the driving capability of automated vehicles (AVs) [1]. For validity and effectiveness, the test scenarios should be concretized with the road geometry [2-5], traffic situations, and microscopic vehicle maneuvers in detail, and should represent complicated road traffic conditions as well as dangerous vehicle maneuvers. Among the many types of road sections, urban roads are known to be the most complicated because of various traffic controls, many entry/exit points, and a variety of road users [6]; such conditions are a threat to AVs and degrade the performance of automated driving. There are various dangerous traffic situations on urban roads and AVs should be tested under these conditions to ensure that they can be used on the roads.

However, it is challenging to generate representative test scenarios because there are many traffic situations on urban roads [7]. Various data sources such as traffic cameras and AVs sensor [8] datasets can be used to derive various situations on urban roads; however, such datasets are too extensive to analyze $[9,10]$ and extracting only unsafe traffic conditions is more challenging [11]. Traffic accident data [12] are useful for extracting unsafe traffic conditions, critical objects, and behaviors that cause accidents. In particular, traffic accident data include details of the road types and geometric characteristics, traffic conditions, and vehicle maneuvers before and during an accident, which can be used to construct test scenarios.

With the advantage of traffic accident data in extracting test scenarios, this study aims to develop a test scenario-mining methodology using a natural language processing technique (NLP). That is, the NLP technique is used to analyze the texts [13] in accident 
investigation reports and extract important elements (e.g., road types and geometric characteristics, traffic conditions, and vehicle maneuvers) that cause an accident. It should be noted that the traffic accident report includes both structured data consisting of code numbers and text-based data written by police officers.

To carry out this study, Section 2 reviews previous studies on automated vehicle test scenarios and application of NLP technique in traffic engineering field. Section 3 presents the premise of the automated vehicle driving capability and operational design domain (ODD) [14] used in this study. Section 4 presents a test scenario-mining methodology for generating automated vehicle safety assessment scenarios from traffic accident data. Section 5 presents the results of the derived features and functional scenarios mined by both the proposed methodology and traffic accident data collected from urban arterial roads in Korea. Also, to verify the derived functional scenarios, a verification process is conducted Section 6 presents the conclusions. Section 7 presents future research tasks.

\section{Literature Review}

To develop safety assessment scenarios for AVs, prior research related to the safety assessment of AVs was reviewed in this study. Choi and Lim [15] developed a scenario for the AEBS test using National Highway Traffic Safety Administration (NHTSA) traffic accident data and an automotive collision case catalog, which is a type of Korean traffic accident data source. A PC-Crash simulation was used for the data analysis. Additionally, traffic accident data were analyzed to develop scenarios according to the road types and traffic accident types. From the analysis, the road types where traffic accidents occurred in Korea were divided into five types and the collision types were divided into six types. Moreover, based on the analysis results, ten accidents at intersections and five accidents on road sections were classified. Finally, a total of 3960 AEBS scenarios were developed using the velocity, angle, and offset at the collision. Zhu et al. [16] proposed a method that uses optimization searching to generate parameters for automated vehicle risk scenarios. The proposed method included five modules. The five modules are composed of an exploration and exploitation module, moving probability determination, step size determination, a memory function module, and a result analysis module. The proposed method could quickly find the risk parameter space in a given logical scenario. Nalic et al. [17] developed a co-simulation framework to develop scenarios for the evaluation and verification of AVs. A method combining the IPG CarMaker and PTV VISSIM was proposed for co-simulation development. In the proposed method, data were processed for every simulation cycle and a new scenario was constructed. All tests that ran data were saved with the relevant scenarios, confirming that real traffic scenarios can be created indefinitely and used for testing. Holland and Sargolzaei [18] proposed a methodology to create and verify automated vehicle scenarios based on actual automated vehicle traffic accidents. The proposed method makes the design of a virtual road possible using the accident location of an automated vehicle and map data based on the accident location. Moreover, an accident scenario was generated using automated vehicle accident description data using a natural language processing (NLP) technique. The generated scenario included the actual characteristics of the road, such as the curvature and number of lanes. So et al. [19] presented a methodology for developing automated vehicle test scenarios. Based on the big data technique, the descriptions of the crash data were analyzed with a C\# -based automated analysis program and an automated vehicle test scenario was verified with a combination of 19 frequently mentioned keywords. As a result, scenarios were derived from road and intersection sections. As a target object, this research study developed only vehicle-to-vehicle scenarios. Menzel et al. [20] presented the terminology and experimental process of an automated vehicle experiment scenario. The concept and experimental stages of functional, logical, and concrete scenarios were defined according to the level of abstraction of the scenario contents. Waymo [21] was used to test a total of 47 functions by adding 19 behavioral functions to the 28 behavioral functions that AVs should perform, as recommended by the NHTSA. A scenario was derived and tested using an automated vehicle developed 
by Waymo. Additionally, to test the collision avoidance capability, based on the NHTSA's pre-collision scenarios, 28 scenarios in four categories were derived and the scenarios were added based on the experimental results in the future. Chen and Kloul [22] proposed an approach to automatically generate use cases of AVs for highways. The proposed approach combined three ontologies as a knowledge base for the generation of highway scenarios, including highway, weather, and vehicle. Relationships and rules, such as traffic regulation, were expressed using first order logic. Ghodsi et al. [23] presented a method of generating and characterizing scenarios for AV safety testing. In the study, the authors used next generation simulation (NGSIM) data to characterize the real driving and adversarial scenarios generated in the simulation. The proposed methodology could generate 240 unsafe scenarios per hour. Riedmaier et al. [24] surveyed a scenario-based safety assessment of AVs. In this research study, there were two ways of scenario generation and extraction that were knowledge-based and data-driven. According to the authors, an infinite number of different scenarios can theoretically occur in real-world traffic, thus finding representative scenarios is important.

Additionally, the present study reviewed research related to NLP technique, which is a method in which computers can understand documents or words written by human languages [25]. NLP is used for traffic engineering area to extract important information for the analysis of traffic accidents and traffic management.

Kamerkar et al. [26] used a text-mining technique to analyze rail accidents in India. This study proposed ensemble methodology using text data and the proposed methodology could automatically discover characteristics of rail accidents. Cheng et al. [27] used a machine learning technique based on NLP to classify accidents in construction site. This study used accident reports to extract relevant knowledge and information, which can be valuable to prevent future accidents in construction projects. Gao et al. [28] developed a verb-based NLP technique using traffic accident data. This study collected and used traffic accident data from the Missouri State Highway Patrol from 19 May to 27 June 2012. As a result of the analysis, it was shown that important information can be extracted for understanding traffic accidents. Ali et al. [29] developed fuzzy ontology and LSTM-based text-mining methodology to monitor the transportation network for assisting travel. This study used an NLP technique to extract relevant features from user-generated text on social media. This study showed that text data generated by social media users can be used for transportation entities or feature extraction. Ali et al. [30] proposed a traffic accident detection and condition analysis framework using social networking data and NLP techniques, such as OLDA, word embedding, and Bi-LSTM. This study showed that the proposed framework can offer detection and analysis that extracts the most valuable traffic information from unstructured data and represents accurately detected and analyzed traffic situations.

\section{Premise of Scenario Development}

3.1. Automated Vehicle Driving Capability

To develop safety assessment scenarios, it is necessary to define the automated vehicle capabilities in advance, such as the automated vehicle level and functions. To this end, the technology level, which is the Society Automotive Engineers (SAE) [31] of the target automated vehicle, was defined.

This study selected level 4 for target automated vehicles, which is a high automation that $\mathrm{AVs}$ can have independently without providing control to the driver in unsafe traffic conditions. However, because the level 4 fully automated vehicle is currently under development, there is a problem in that it is difficult to determine the exact specifications. Thus, the range of capabilities of the automated vehicle was defined. The behavioral competency of the target automated vehicle was defined as stop and go, lane-change, passing through a signalized intersection, and turning an intersection. This automated vehicle is capable of cooperative driving using V2X communication, which leads to the communication and recognition of traffic signals at signalized intersections. It was assumed 
that the automated vehicle defined in this study does not cause malfunction and drives itself by following the given driving rules and laws.

\subsection{Operation Design Domain}

For an automated vehicle to drive properly, it is necessary to set the drivable areas and conditions. Defining the drivable areas and conditions is referred to as ODD. Currently, ODD has various definitions in many international standards. According to the ISO 21448 standard [32], ODD is "the specific conditions under which a given driving automation system is designed to function". Specific conditions include spatial, temporal, and environmental conditions.

In this study, spatial, temporal, and environmental conditions were defined for AVs. The spatial-specific conditions, road type, number of lanes, and speed limit were selected. To define the road type, this research considered continuity with expressways and selected urban arterial roads, including roads and intersections. This is because the urban arterial road is the road where AVs will be introduced, next to the expressway, after the expansion to other roads to reach the destination. Figure 1 shows the concept of extended odd from expressway to urban arterial roads.

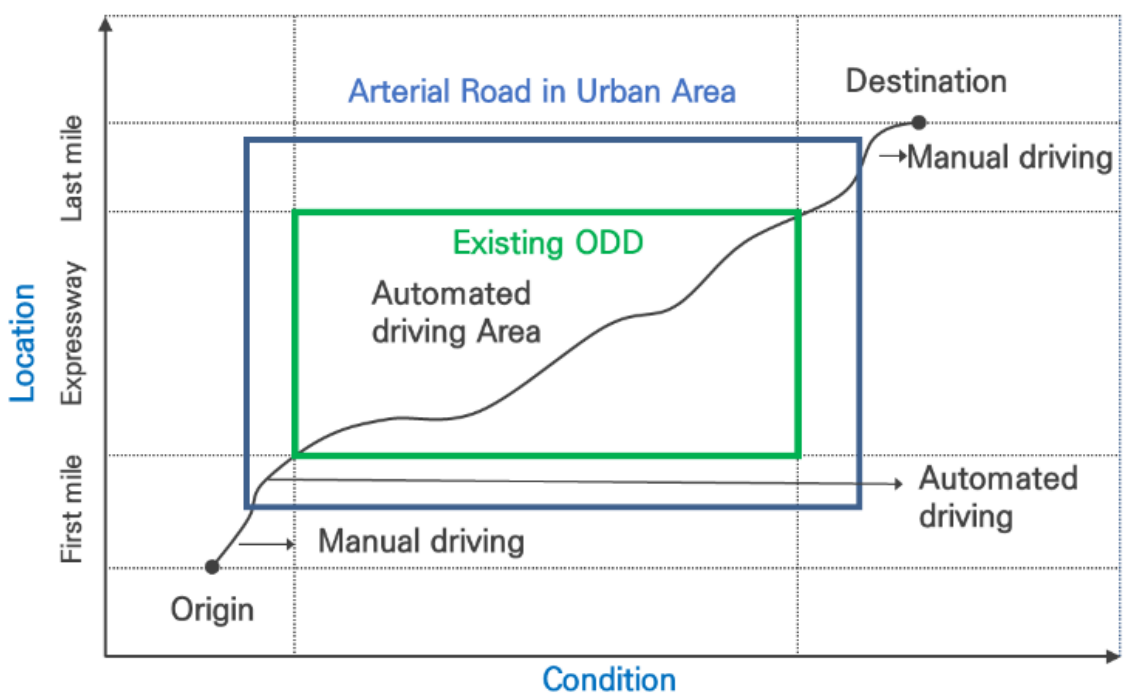

Figure 1. Concept of extended ODD to urban arterial roads.

To find specific elements, such as the number of lanes and speed limits, a Korean national standard node link GIS map was used. Next, the temporal condition was defined as 7:00-18:00 h, which is in the daytime. Finally, the weather was considered the environmental conditions and defined as sunny. The defined ODD is expressed as shown in Table 1.

Table 1. Defined ODD for urban arterial roads in Korea.

\begin{tabular}{ccc}
\hline Categories & Items & ODDs \\
\hline \multirow{2}{*}{ Spatial condition } & Road type & $\begin{array}{c}\text { Urban arterial road including } \\
\text { signalized intersection in Korea }\end{array}$ \\
\cline { 2 - 3 } & Number of lanes & $4 \sim 8$ \\
\cline { 2 - 3 } & Speed limit & $30 \sim 90$ \\
\hline Temporal condition & Time & Daytime \\
\hline Environmental condition & Weather & Sunny \\
\hline
\end{tabular}




\section{Methodology}

\subsection{Overview}

This study proposes a scenario-mining methodology for the automated vehicle safety assessment from traffic accident data using an NLP technique. Considering the traffic accident data includes the 'accident situation description' described in the text, it is possible to understand the traffic accident situation. In this study, automated vehicle scenarios were developed by extracting traffic accidents that occurred on arterial roads in urban areas and using the proposed methodology. The proposed methodology consists of six steps: data collection, data extraction, data preprocessing, feature extraction, feature categorization by object, and scenario-mining. Figure 2 shows the structure of the proposed research methodology.
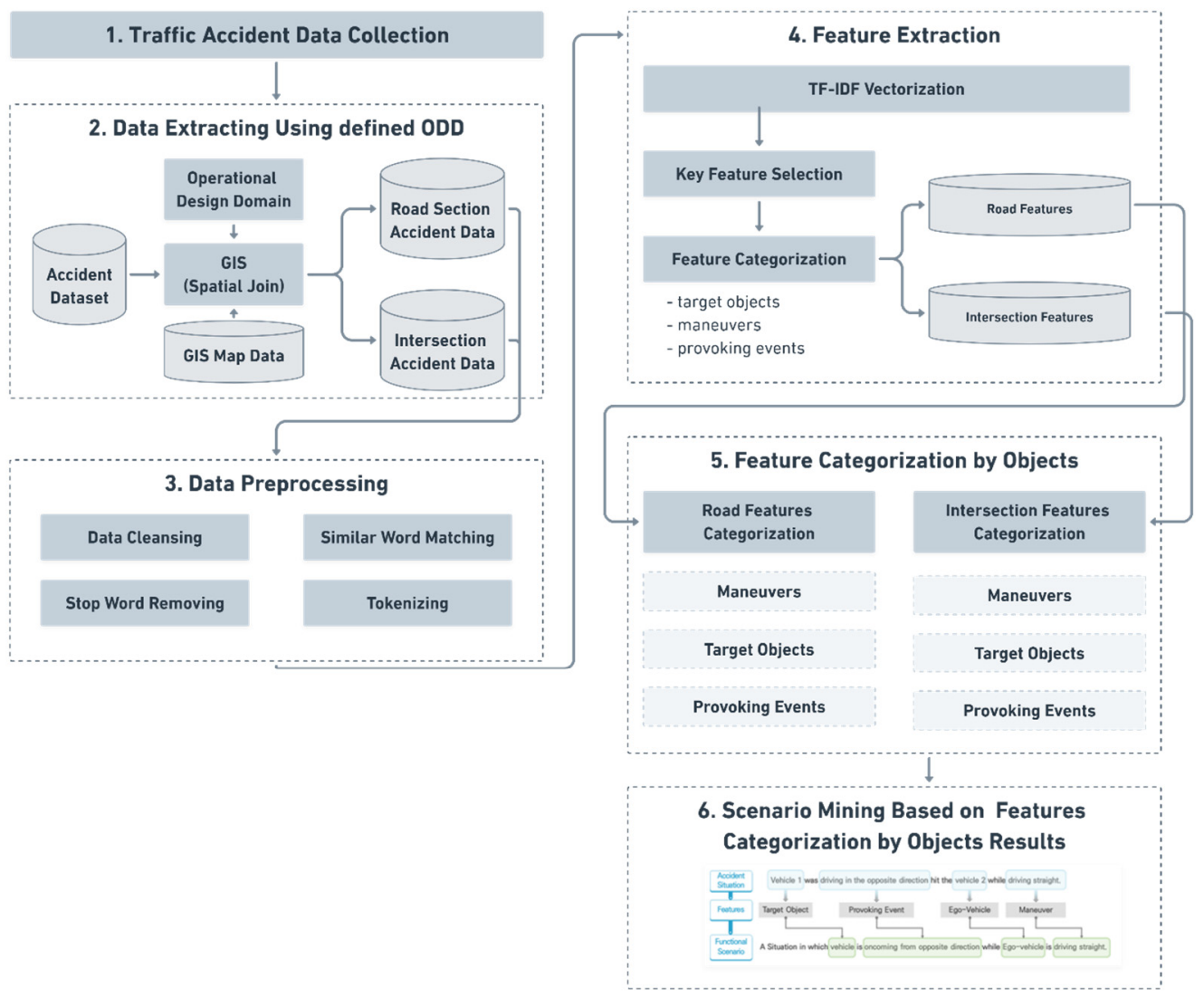

Figure 2. Architecture of scenario-mining process.

\subsection{Data Collection}

To develop a scenario for the automated vehicle safety assessment, this study utilized general automobile traffic accident data managed by the Korean National Policy Agency (KNPA). To assess the safety of AVs, it is best to use AV traffic accident data. However, the existing AV traffic accident data remains insufficient to generate scenarios. In addition, in mixed traffic conditions with human-based objects such as vehicles, pedestrians, motorcycles, and bicycles, AVs would encounter dangerous situations caused by human-based objects, as general vehicles have encountered as well. Thus, KNPA automobile traffic accident data could be an alternative to develop AV scenarios.

The KNPA traffic accident data include various data such as time, location (GPS coordinates), accident type, vehicle type, and accident situation descriptions. Therefore, it is possible to analyze the object and situation that caused the traffic accident at the time. Particularly, the 'accident situation description' describes the situation in the event of a traffic accident, written in text. In this study, 223,552 traffic accident data from 2014 were collected nationwide to perform the scenario-mining. 
To extract the traffic accident data that occurred on urban arterial roads in Korea, it was necessary to extract only the relevant traffic accidents from the collected data. As the traffic accident data of the KNPA include GPS coordinates, it is possible to extract the relevant traffic accident by performing spatial join using GIS software. Therefore, traffic accidents that occurred in urban arterial roads and at intersections were extracted through spatial join with the accident data, defined ODD, and GIS map. In this study, spatial join was performed using ArcGIS 10.3, a representative GIS tool. As a result of extracting data through spatial join, 2824 road sections and 4166 intersection sections were extracted.

\subsection{Data Preprocessing}

To utilize text data, which is an "accident situation description" from the accident data, preprocessing is essential. To this end, the Python 3.7 and Mecab library, which is the predominant Korean natural language process library, was utilized for data preprocessing.

In this study, we reviewed and selected a text data preprocessing technique. Ten preprocessing techniques are frequently used [33]. The data preprocessing has four steps: data cleansing, similar word matching, stop word removal, and tokenization. In the data cleansing step, this study removed text such as punctuation marks, special characters, numbers, etc., which cannot grasp the meaning from the data. A similar word matching step could address synonyms because different people may use different words to record accident situation descriptions. A stop word removal step was then performed. Stop words are common words with no semantics and do not aggregate relevant information to the task, such as "the" and "a" [33]. Lastly, the tokenization step divides each accident situation description sentence into token units, which are small chunks such as words and attached parts of speech. In particular, in this study, among several parts of speech, nouns, including compound nouns, were used.

\subsection{Feature Extraction}

To extract features, which are meaningful words, from text data, the feature extraction process is essential. To select the relevant feature extraction method, this study reviewed feature extraction methods. There are four feature extraction methods including bag-ofwords, the term frequency-inverse document frequency (TF-IDF) model, and Word2Vec, which is mainly used in the NLP [34].

This study selected the TF-IDF model, which is the most widely used in NLP and has the advantage of expressing the relative importance of each word in an individual document. Additionally, the TF-IDF model is able to provide weight to words that appear frequently throughout the document rather than simply applying weight by the frequency [35]. Equation (1) is a TF-IDF model.

$$
T F-I D F_{(w, d)}=T F_{(w, d)} \times \log \left(\frac{n}{D F_{(w)}}\right)
$$

where $T F_{(w, d)}=$ number of words, $w$, in documents, $d ; n=$ number of total documents; and $D F_{(w)}=$ number of documents including words, $w$.

Using the TF-IDF model, this study derived features and TF-IDF values from the collected data of the urban arterial roads and intersections. To derive more meaningful features, trivial features such as the area names, proper nouns, and vehicle names were removed. After that, features were categorized into target objects, maneuvers, provoking events, and so on to determine the meaning of the features.

\subsection{Feature Categorization by Objects}

Each of the derived features has its own meaning but there is a limitation in explaining the corresponding accident situation that contains the features. However, although features also occur individually, they tend to occur together in a specific object. For example, in a traffic accident situation related to vehicle-to-vehicle accidents, a collision due to a 
stop may occur, but crosswalk-crossing does not occur. Therefore, feature categorization was performed by objects such as vehicles, pedestrians, bicycles, and motorcycles. The features by object were categorized by the accident location, maneuver, target object, and provoking event.

\subsection{Generation of Functional Scenarios}

To develop scenarios for the automated vehicle safety assessment, this study utilized the functional scenario concept proposed by the Pegasus project. This is a project for the establishment of generally accepted quality criteria, tools, and methods, as well as scenarios and situations, for the release of highly automated driving functions, organized under the initiative of the German Federal Ministry for Economic Affairs and Energy [36,37]. A functional scenario is one in which road sections, fixed and dynamic elements, and situations are described in natural language with a high level of abstraction [38].

To develop functional scenarios, this study used previously derived feature categories, maneuvers, target objects, and provoking events, and developed a scenario development system. Specifically, in an accident situation, the object causing the accident was defined as the target object. An action that caused an accident was defined as a provoking event. The victim vehicle was defined as an ego-vehicle and the driving situation at the time was defined as the maneuver of the ego-vehicle. For example, in the situation 'Vehicle 1 which was driving in the opposite direction hit vehicle 2 which was driving straight', the features are extracted such that 'vehicle 1 ' is the target object, 'driving in the opposite direction' is the provoking event of the target object, 'vehicle 2 ' is the victim vehicle defined as the ego-vehicle, and 'driving straight' is the maneuver of the ego-vehicle. Finally, the features were constructed into a functional scenario. Figure 3 depicts the procedure in which the accident data were composed into a functional scenario. It should also be noted that this study includes only the traffic interactions and situations in which AVs are spontaneously involved, while the situations in which normal vehicles crash into the back of AVs, which is unavoidable from the view of AVs, are excluded.

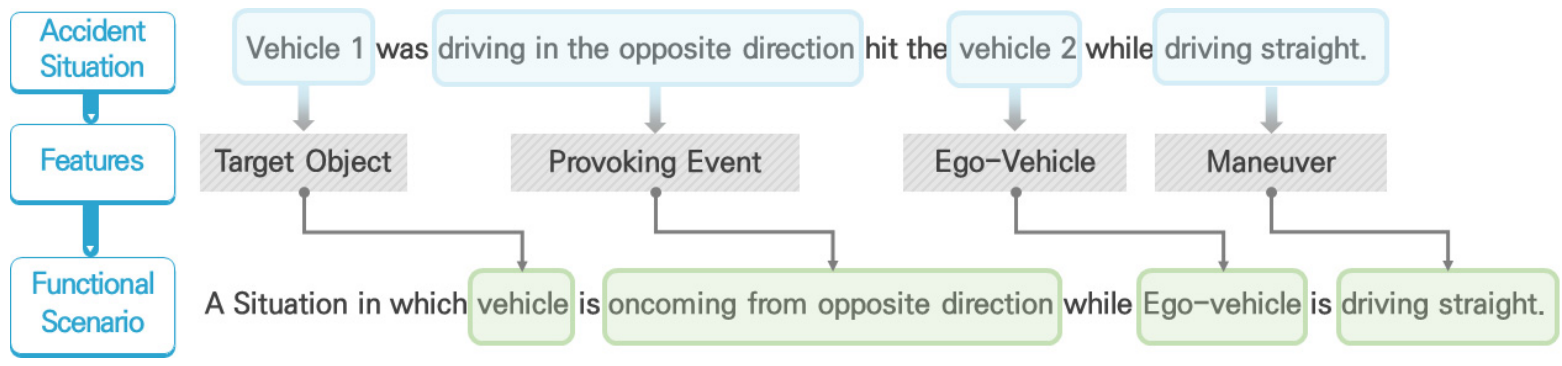

Figure 3. Functional scenario development procedure.

\section{Results}

\subsection{Key Feature Extraction Results}

The features of the road sections and intersections were extracted using the Python and TF-IDF model. For the road sections, 2811 features were extracted. However, since there were insignificant features that could not depict accident situations, such as specific location (municipality), building name, subway station name, vehicle's brand/maker, and so on (e.g., Seoul, apartment, Sonata, Sadang station, etc.), postprocessing was performed to remove such insignificant features. After postprocessing, fifteen main features were obtained and categorized into object, maneuver, and provoking events. Consequently, vehicles, pedestrians, bicycles, and motorcycles were extracted as the objects; in the case of maneuvering, driving straight, and lane-change, crossing, stop, and U-turn were obtained as the provoking events. Table 2 shows obtained features on the road section. 
Table 2. Obtained features on the road section.

\begin{tabular}{|c|c|c|c|}
\hline Section & Objects & Maneuver & Provoking Events \\
\hline \multirow{10}{*}{ Road } & $\begin{array}{l}\text { Vehicle } \\
(1131.54)\end{array}$ & $\begin{array}{c}\text { Driving Straight } \\
(527.30)\end{array}$ & $\begin{array}{c}\text { Lane-change } \\
(254.64)\end{array}$ \\
\hline & $\begin{array}{l}\text { Pedestrian } \\
\quad(143.36)\end{array}$ & - & $\begin{array}{l}\text { Crossing } \\
(135.75)\end{array}$ \\
\hline & $\begin{array}{l}\text { Bicycle } \\
(116.34)\end{array}$ & - & $\begin{array}{l}\text { Stopping } \\
(93.19)\end{array}$ \\
\hline & $\begin{array}{c}\text { Motorcycle } \\
\quad(111.86)\end{array}$ & - & $\begin{array}{l}\text { U-turn } \\
(82.33)\end{array}$ \\
\hline & - & - & $\begin{array}{c}\text { Abrupt stopping } \\
(44.10)\end{array}$ \\
\hline & - & - & $\begin{array}{c}\text { Driving over centerline } \\
\text { (37.43) }\end{array}$ \\
\hline & - & - & $\begin{array}{c}\text { Walking } \\
(35.12)\end{array}$ \\
\hline & - & - & $\begin{array}{c}\text { Abrupt lane-change } \\
(31.52)\end{array}$ \\
\hline & - & - & $\begin{array}{c}\text { Jaywalking } \\
(19.09)\end{array}$ \\
\hline & - & - & $\begin{array}{c}\text { Reversing } \\
\quad(7.48)\end{array}$ \\
\hline
\end{tabular}

(): TF-IDF value.

For the intersection sections, 4096 features were extracted and a total of 15 features were obtained by removing the insignificant features. The main features were categorized by object, maneuver, and provoking event. Among the obtained main features, the object items were extracted as vehicles, bicycles, motorcycles, and pedestrians, and in the case of maneuvers, this research extracted driving straight, left-turn, and right-turn. Stopping, lanechange, crossing, and violating traffic signals were obtained as the provoking situations. Table 3 shows obtained features on the intersection section.

Table 3. Obtained features on the intersection section.

\begin{tabular}{|c|c|c|c|}
\hline Section & Objects & Maneuvers & Provoking Events \\
\hline \multirow{8}{*}{ Intersection } & $\begin{array}{l}\text { Vehicle } \\
(1583.78)\end{array}$ & $\begin{array}{c}\text { Driving straight } \\
(769.30)\end{array}$ & $\begin{array}{l}\text { Stopping } \\
(291.44)\end{array}$ \\
\hline & $\begin{array}{c}\text { Bicycle } \\
(190.394)\end{array}$ & $\begin{array}{l}\text { Left-turn } \\
(413.54)\end{array}$ & $\begin{array}{c}\text { Lane-change } \\
(214.32)\end{array}$ \\
\hline & $\begin{array}{l}\text { Motorcycle } \\
(171.20)\end{array}$ & $\begin{array}{l}\text { Right-turn } \\
(311.90)\end{array}$ & $\begin{array}{l}\text { Crossing } \\
(213.67)\end{array}$ \\
\hline & $\begin{array}{l}\text { Pedestrian } \\
\quad(166.60)\end{array}$ & - & $\begin{array}{c}\text { Violating traffic signal } \\
(141.34)\end{array}$ \\
\hline & - & - & $\begin{array}{l}\text { U-turn } \\
(121.97)\end{array}$ \\
\hline & - & - & $\begin{array}{l}\text { Walking } \\
(43.00)\end{array}$ \\
\hline & - & - & $\begin{array}{l}\text { Abrupt stopping } \\
(32.98)\end{array}$ \\
\hline & - & - & $\begin{array}{c}\text { Jaywalking } \\
(14.68)\end{array}$ \\
\hline
\end{tabular}

(): TF-IDF value. 


\subsection{Feature Categorization by Objects}

To analyze the obtained features in detail, the main features were extracted by classifying them according to the target object. To obtain the corresponding word from the text analysis, meaningful words were obtained simultaneously rather than single words alone. In the case of a vehicle in the road section, lane-change, stopping, U-turn, etc., were obtained. The driving over centerline and reversing were obtained. Crossing, walking, and jaywalking were obtained for the pedestrians. In the case of walking, it means walking on the road and not walking on the pedestrian. For motorcycles, lane changes, crossings, U-turns, and stops were obtained. In the case of bicycles, crossing, reversing, and straight driving were obtained. Table 4 shows obtained features in the road section by objects.

Table 4. Obtained features on the road section by objects.

\begin{tabular}{|c|c|c|c|c|}
\hline \multirow{2}{*}{ Location } & \multirow{2}{*}{ Maneuver } & \multirow{2}{*}{ Objects } & \multicolumn{2}{|c|}{ Provoking Events } \\
\hline & & & Features & TF-IDF \\
\hline \multirow{17}{*}{ Road } & \multirow{17}{*}{ Driving Straight } & \multirow{7}{*}{ Vehicle } & Lane-change & 292.67 \\
\hline & & & Stopping & 104.04 \\
\hline & & & U-turn & 78.83 \\
\hline & & & Abrupt stopping & 48.77 \\
\hline & & & Driving over centerline & 36.43 \\
\hline & & & Abrupt lane-change & 34.08 \\
\hline & & & Reversing & 5.27 \\
\hline & & \multirow{3}{*}{ Pedestrian } & Crossing & 51.34 \\
\hline & & & Walking & 18.45 \\
\hline & & & Jaywalking & 11.96 \\
\hline & & \multirow{4}{*}{ Motorcycle } & Lane-change & 23.67 \\
\hline & & & Crossing & 11.54 \\
\hline & & & U-turn & 7.58 \\
\hline & & & Stopping & 4.65 \\
\hline & & \multirow{3}{*}{ Bicycle } & Crossing & 29.25 \\
\hline & & & Driving straight & 12.13 \\
\hline & & & Reversing & 1.36 \\
\hline
\end{tabular}

In the case of the intersection sections, the maneuver of the vehicle was obtained as driving straight, left-turn, and right-turn. From the object analysis, in the case of a vehicle, stopping, lane-change, violating traffic signal, U-turn, and abrupt stopping were obtained.

Crossing, walking, and jaywalking were obtained for pedestrians. In the case of motorcycles, lane-change, stop, violating traffic signals, and crossings were obtained. In the case of bicycles, crossing, reversing, stopping, and violating traffic signals were obtained. Table 5 shows obtained features in the intersection sections by objects.

\subsection{Scenario Development Results}

Using the features obtained from the objects and the scenario development system, functional scenarios of road sections and intersections of urban arterial roads were developed. For the road section, a total of 16 scenarios were derived. All derived scenarios for the road section are presented in Appendix A. In the case of a vehicle as a target object, seven scenarios were developed, as presented in Table A1. In the case of a pedestrian as a target object, three scenarios were derived, as presented in Table A2. In the case of a motorcycle as a target object, three scenarios were derived, as presented in Table A3. In the 
case of a bicycle as a target object, three scenarios were derived, as presented in Table A4. Table 6 shows examples of developed functional scenarios in road sections.

Table 5. Obtained features at the intersection sections by objects.

\begin{tabular}{|c|c|c|c|c|}
\hline \multirow{2}{*}{ Location } & \multirow{2}{*}{ Maneuvers } & \multirow{2}{*}{ Objects } & \multicolumn{2}{|c|}{ Provoking Events } \\
\hline & & & Features & TF-IDF \\
\hline \multirow{18}{*}{ Intersection } & \multirow{2}{*}{ Driving straight } & \multirow{5}{*}{ Vehicle } & Stopping & 396.21 \\
\hline & & & Lane-change & 294.10 \\
\hline & \multirow{2}{*}{ Left-turn } & & Violating traffic signal & 169.77 \\
\hline & & & U-turn & 125.03 \\
\hline & Right-turn & & Abrupt stopping & 40.44 \\
\hline & \multirow{2}{*}{ Driving straight } & \multirow{5}{*}{ Pedestrian } & Crossing & 104.26 \\
\hline & & & Walking & 41.03 \\
\hline & \multirow[t]{2}{*}{ Left-turn } & & & \\
\hline & & & Stopping & 20.91 \\
\hline & Right-turn & & Jaywalking & 15.57 \\
\hline & \multirow{2}{*}{ Driving straight } & \multirow{5}{*}{ Motorcycle } & Lane-change & 26.54 \\
\hline & & & Stopping & 20.45 \\
\hline & \multirow[t]{2}{*}{ Left-turn } & & & \\
\hline & & & Violating traffic signal & 20.20 \\
\hline & Right-turn & & Crossing & 16.16 \\
\hline & Driving straight & \multirow{3}{*}{ Bicycle } & Crossing & 19.03 \\
\hline & Left-turn & & Stopping & 2.52 \\
\hline & Right-turn & & Violating traffic signal & 1.64 \\
\hline
\end{tabular}

For the intersection sections, a total of 38 scenarios were obtained. All derived scenarios for the intersection sections are presented in Appendix B. In the case of a vehicle as a target object, sixteen scenarios were developed, as presented in in Table A5. In the case of a pedestrian as a target object, three scenarios were developed, as presented in Table A6. In the case of a motorcycle as a target object, sixteen scenarios were developed, as presented in Table A7. In the case of a bicycle as a target object, three scenarios were developed, as presented in Table A8. Table 7 presents an example of developed functional scenarios in intersection sections. 
Table 6. Examples of developed functional scenarios on road sections.

\begin{tabular}{|c|c|c|c|c|}
\hline No. & Depictions & Maneuvers & Provoking Events & Functional Scenarios \\
\hline 1 & 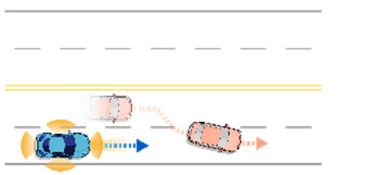 & Driving straight & Lane-change & $\begin{array}{l}\text { Ego-vehicle is driving straight at road section. } \\
\text { Target object (Vehicle) is lane-changing into } \\
\text { ego-vehicle's driving lane ahead. }\end{array}$ \\
\hline 2 & 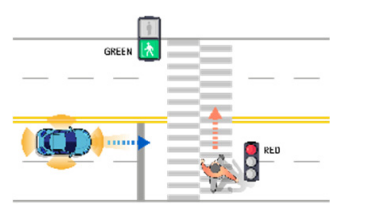 & Driving straight & Crossing & $\begin{array}{l}\text { Ego-vehicle is driving straight at road section. } \\
\text { Target object (Pedestrian) is crossing ahead. }\end{array}$ \\
\hline 3 & $\begin{array}{l}------- \\
-6 \frac{1}{10}--\end{array}$ & Driving straight & Lane-change & $\begin{array}{l}\text { Ego-vehicle is driving straight at road section. } \\
\text { Target object (Motorcycle) is lane-changing } \\
\text { into ego-vehicle's driving lane. }\end{array}$ \\
\hline 4 & $\begin{array}{l}------- \\
-\cos ----\end{array}$ & Driving straight & Driving straight & $\begin{array}{l}\text { Ego-vehicle is driving straight at road section. } \\
\text { Target object (Bicycle) is driving straight into } \\
\text { ego-vehicle's lane ahead. }\end{array}$ \\
\hline
\end{tabular}

Table 7. Examples of developed functional scenarios at intersection sections.

\begin{tabular}{llccc}
\hline No. Depictions & Maneuvers & Provoking Events & Functional Scenarios \\
\hline 1 & & Driving straight & Violating traffic signal & $\begin{array}{c}\text { Ego-vehicle is driving straight on the right of } \\
\text { way at signalized intersection. Target object } \\
\text { (Vehicle) that violates a traffic signal from the } \\
\text { left is driving straight. }\end{array}$ \\
\end{tabular}

2 䡞- Driving straight Jaywalking

3

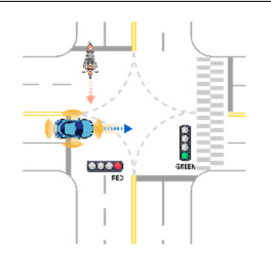

Driving straight Violating traffic signal

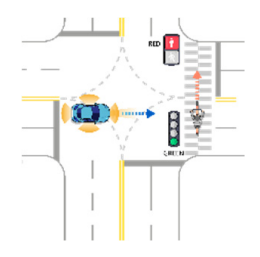

4

Ego-vehicle is driving straight on the right of way at signalized intersection. Target object (Pedestrian) is jaywalking ahead at crosswalk.
Ego-vehicle is driving straight on the right of way at signalized intersection. Target object (Motorcycle) that violates a traffic signal from the left is driving straight. 


\subsection{Verification of the Resulting Scenarios}

To verify the derived functional scenario, a verification process was performed. This research verified the number of functional scenarios that occur in real traffic accidents in road and intersections. For road sections, the functional scenarios developed in this study accounted for $43.69 \%$ of the actual traffic accidents. Vehicle-to-vehicle functional scenarios accounted for $39.35 \%$ of the actual traffic accidents in road sections. The ratio of vehicle-to-vehicle functional scenarios from real accident data in road sections is shown in Table 8.

Table 8. Ratio of vehicle-to-vehicle functional scenarios from real accident data on road sections.

\begin{tabular}{|c|c|c|c|}
\hline No. & Provoking Events & Functional Scenarios & Ratios \\
\hline 1 & Lane-change & $\begin{array}{l}\text { Ego-vehicle is driving straight at road section. Target object } \\
\text { (Vehicle) is lane-changing into ego-vehicle's driving lane ahead. }\end{array}$ & $22.70 \%$ \\
\hline 2 & Abrupt lane-change & $\begin{array}{l}\text { Ego-vehicle is driving straight at road section. Target object } \\
\text { (Vehicle) is suddenly lane-changing into ego-vehicle's driving } \\
\text { lane ahead. }\end{array}$ & $2.48 \%$ \\
\hline 3 & Stopping & $\begin{array}{l}\text { Ego-vehicle is driving straight at road section. Target object } \\
\text { (Vehicle) is stopping in ego-vehicle's driving lane ahead. }\end{array}$ & $5.35 \%$ \\
\hline 4 & U-turn & $\begin{array}{l}\text { Ego-vehicle is driving straight at road section. Target object } \\
\text { (Vehicle) is making a U-turn into ego-vehicle's driving lane ahead. }\end{array}$ & $4.25 \%$ \\
\hline 5 & Abrupt stopping & $\begin{array}{l}\text { Ego-vehicle is driving straight at road section. Target object } \\
\text { (Vehicle) is suddenly stopping in ego-vehicle's driving lane ahead. }\end{array}$ & $2.37 \%$ \\
\hline 6 & Driving over centerline & $\begin{array}{l}\text { Ego-vehicle is driving straight at road section. Target object } \\
\text { (Vehicle) is driving over centerline into ego-vehicle's driving lane. }\end{array}$ & $2.02 \%$ \\
\hline 7 & Reversing & $\begin{array}{l}\text { Ego-vehicle is driving straight at road section. Target object } \\
\text { (Vehicle) is reversing into ego-vehicle's driving lane. }\end{array}$ & $0.18 \%$ \\
\hline
\end{tabular}

Regarding vehicle-to-pedestrian functional scenarios, they account for $2.10 \%$ of the actual traffic accidents in road sections as shown in Table 9.

Table 9. Ratio of vehicle-to-pedestrian functional scenarios from real accident data on road sections.

\begin{tabular}{cccc}
\hline No. & Provoking Events & Functional Scenarios & Ratios \\
\hline 1 & Crossing & $\begin{array}{c}\text { Ego-vehicle is driving straight at road section. Target object } \\
\text { (Pedestrian) is crossing ahead. }\end{array}$ & $1.74 \%$ \\
\hline 2 & Walking & $\begin{array}{c}\text { Ego-vehicle is driving straight at road section. Target object } \\
\text { (Pedestrian) is walking in lane ahead. }\end{array}$ & $0.04 \%$ \\
\hline 3 & Jaywalking & $\begin{array}{c}\text { Ego-vehicle is driving straight at road section. Target object } \\
\text { (Pedestrian) is jaywalking ahead. }\end{array}$ & $0.32 \%$ \\
\hline
\end{tabular}

Regarding vehicle-to-motorcycle functional scenarios, they accounted for $1.07 \%$ of the actual traffic accidents in road sections as shown in Table 10.

Table 10. Ratio of vehicle-to-motorcycle functional scenarios from real accident data on road sections.

\begin{tabular}{cccc}
\hline No. & Provoking Events & Functional Scenarios & Ratios \\
\hline 1 & Lane-change & $\begin{array}{c}\text { Ego-vehicle is driving straight at road section. Target object } \\
\text { (Motorcycle) is lane-changing into ego-vehicle's driving lane. }\end{array}$ & $0.71 \%$ \\
\hline 2 & U-turn & $\begin{array}{c}\text { Ego-vehicle is driving straight at road section. Target object } \\
\text { (Motorcycle) is making a U-turn into ego-vehicle's driving lane. }\end{array}$ & $0.18 \%$ \\
\hline 3 & Stopping & $\begin{array}{c}\text { Ego-vehicle is driving straight at road section. Target object } \\
\text { (Motorcycle) is stopping in ego-vehicle's driving lane ahead. }\end{array}$ & $0.18 \%$ \\
\hline
\end{tabular}


Regarding vehicle-to-bicycle functional scenarios, they accounted for $1.17 \%$ of the actual traffic accidents in road sections as shown in Table 11.

Table 11. Ratio of vehicle-to-bicycle functional scenarios from real accident data on road sections.

\begin{tabular}{|c|c|c|c|}
\hline No. & Provoking Events & Functional Scenarios & Ratios \\
\hline 1 & Crossing & $\begin{array}{l}\text { Ego-vehicle is driving straight at road section. Target object } \\
\text { (Bicycle) is crossing ahead. }\end{array}$ & $0.96 \%$ \\
\hline 2 & Driving straight & $\begin{array}{l}\text { Ego-vehicle is driving straight at road section. Target object } \\
\text { (Bicycle) is driving straight into ego-vehicle's lane ahead. }\end{array}$ & $0.14 \%$ \\
\hline 3 & Reversing & $\begin{array}{l}\text { Ego-vehicle is driving straight at road section. Target object } \\
\text { (Bicycle) is reversing into ego-vehicle's driving lane. }\end{array}$ & $0.07 \%$ \\
\hline
\end{tabular}

For the intersection sections, the developed functional scenarios in this study were found to account for $27.63 \%$ of the actual traffic accidents. Regarding vehicle-to-vehicle functional scenarios, they accounted for $19.8 \%$ of the actual traffic accidents at intersection sections. Table 12 shows ratio of vehicle-to-vehicle functional scenarios from real accident data at intersection sections.

Table 12. Ratio of vehicle-to-vehicle functional scenarios from real accident data at intersection sections.

\begin{tabular}{|c|c|c|c|}
\hline No. & Provoking Events & Functional Scenarios & Ratios \\
\hline 1 & Violating traffic signal & $\begin{array}{c}\text { Ego-vehicle is driving straight on the right of way at signalized } \\
\text { intersection. Target object (Vehicle) that violates a traffic signal from } \\
\text { the left is driving straight. }\end{array}$ & $1.01 \%$ \\
\hline 2 & Violating traffic signal & $\begin{array}{l}\text { Ego-vehicle is driving straight on the right of way at signalized } \\
\text { intersection. Target object (Vehicle) that violates a traffic signal from } \\
\text { the right is driving straight. }\end{array}$ & $1.01 \%$ \\
\hline 3 & Violating traffic signal & $\begin{array}{l}\text { Ego-vehicle is driving straight on the right of way at signalized } \\
\text { intersection. Target object (Vehicle) that violates a traffic signal from } \\
\text { the other side is turning right. }\end{array}$ & $2.90 \%$ \\
\hline 4 & Violating traffic signal & $\begin{array}{l}\text { Ego-vehicle is driving straight on the right of way at signalized } \\
\text { intersection. Target object (Vehicle) that violates a traffic signal from } \\
\text { the other side is turning left. }\end{array}$ & $1.03 \%$ \\
\hline 5 & Lane-change & $\begin{array}{l}\text { Ego-vehicle is driving straight on the right of way at signalized } \\
\text { intersection. Target object (Vehicle) is changing lanes into } \\
\text { ego-vehicle's lane in the same direction. }\end{array}$ & $1.90 \%$ \\
\hline 6 & Stopping & $\begin{array}{c}\text { Ego-vehicle is driving straight on the right of way at signalized } \\
\text { intersection. Target object (Vehicle) is stopping in front of } \\
\text { ego-vehicle in the same direction. }\end{array}$ & $1.61 \%$ \\
\hline 7 & Violating traffic signal & $\begin{array}{c}\text { Ego-vehicle is turning left on the right of way at signalized } \\
\text { intersection. Target object (Vehicle) that violates a traffic signal from } \\
\text { the left is driving straight. }\end{array}$ & $1.90 \%$ \\
\hline 8 & Violating traffic signal & $\begin{array}{l}\text { Ego-vehicle is turning left on the right of way at signalized } \\
\text { intersection. Target object (Vehicle) that violates a traffic signal from } \\
\text { the right is driving straight. }\end{array}$ & $1.90 \%$ \\
\hline 9 & Violating traffic signal & $\begin{array}{c}\text { Ego-vehicle is turning left on the right of way at signalized } \\
\text { intersection. Target object (Vehicle) that violates a traffic signal from } \\
\text { the other side is turning left. }\end{array}$ & $1.10 \%$ \\
\hline 10 & Violating traffic signal & $\begin{array}{l}\text { Ego-vehicle is turning left on the right of way at signalized } \\
\text { intersection. Target object (Vehicle) that violates a traffic signal from } \\
\text { the other side is turning right. }\end{array}$ & $1.90 \%$ \\
\hline 11 & Lane-change & $\begin{array}{l}\text { Ego-vehicle is turning left on the right of way at signalized } \\
\text { intersection. Target object (Vehicle) is changing lanes into } \\
\text { ego-vehicle's lane in the same direction. }\end{array}$ & $0.31 \%$ \\
\hline
\end{tabular}


Table 12. Cont.

\begin{tabular}{|c|c|c|c|}
\hline No. & Provoking Events & Functional Scenarios & Ratios \\
\hline 12 & Stopping & $\begin{array}{l}\text { Ego-vehicle is turning left on the right of way at signalized } \\
\text { intersection. Target object (Vehicle) is stopping in front of } \\
\text { ego-vehicle in the same direction. }\end{array}$ & $3.22 \%$ \\
\hline 13 & Violating traffic signal & $\begin{array}{c}\text { Ego-vehicle is turning right on the right of way at signalized } \\
\text { intersection. Target object (Vehicle) that violates a traffic signal from } \\
\text { the other side is driving straight. }\end{array}$ & $0.02 \%$ \\
\hline 14 & Violating traffic signal & $\begin{array}{c}\text { Ego-vehicle is turning right on the right of way at signalized } \\
\text { intersection. Target object (Vehicle) that violates a traffic signal from } \\
\text { the other side is turning left. }\end{array}$ & $0.00 \%$ \\
\hline 15 & Lane-change & $\begin{array}{l}\text { Ego-vehicle is turning right on the right of way at signalized } \\
\text { intersection. Target object (Vehicle) is changing lanes into } \\
\text { ego-vehicle's lane in the same direction. }\end{array}$ & $0.00 \%$ \\
\hline 16 & Stopping & $\begin{array}{l}\text { Ego-vehicle is turning right on the right of way at signalized } \\
\text { intersection. Target object (Vehicle) is stopping in front of } \\
\text { ego-vehicle in the same direction. }\end{array}$ & $0.00 \%$ \\
\hline
\end{tabular}

Regarding vehicle-to-pedestrian functional scenarios, they accounted for $0.58 \%$ of the actual traffic accidents at intersection sections as shown in Table 13.

Table 13. Ratio of vehicle-to-pedestrian functional scenarios from real accident data at intersection sections.

\begin{tabular}{ccccc}
\hline No. & Provoking Events & Functional Scenarios & Ratios \\
\hline 1 & Jaywalking & $\begin{array}{c}\text { Ego-vehicle is driving straight on the right of way at signalized } \\
\text { intersection. Target object (Pedestrian) is jaywalking ahead at crosswalk. }\end{array}$ & $0.24 \%$ \\
\hline 2 & Jaywalking & $\begin{array}{c}\text { Ego-vehicle is turning left on the right of way at signalized intersection. } \\
\text { Target object (Pedestrian) is jaywalking ahead at crosswalk. }\end{array}$ & $0.24 \%$ \\
\hline 3 & Jaywalking & Ego-vehicle is turning right on the right of way at signalized intersection. & $0.10 \%$ \\
\hline
\end{tabular}

Regarding vehicle-to-motorcycle functional scenarios, they accounted for $6.70 \%$ of the actual traffic accidents at intersection sections as shown in Table 14.

Table 14. Ratio of vehicle-to-motorcycle functional scenarios from real accident data at intersection sections.

\begin{tabular}{|c|c|c|c|}
\hline No. & Provoking Events & Functional Scenarios & Ratios \\
\hline 1 & Violating traffic signal & $\begin{array}{l}\text { Ego-vehicle is driving straight on the right of way at signalized } \\
\text { intersection. Target object (Motorcycle) that violates a traffic signal } \\
\text { from the left is driving straight. }\end{array}$ & $0.91 \%$ \\
\hline 2 & Violating traffic signal & $\begin{array}{l}\text { Ego-vehicle is driving straight on the right of way at signalized } \\
\text { intersection. Target object (Motorcycle) that violates a traffic signal } \\
\text { from the right is driving straight. }\end{array}$ & $0.91 \%$ \\
\hline 3 & Violating traffic signal & $\begin{array}{c}\text { Ego-vehicle is driving straight on the right of way at signalized } \\
\text { intersection. Target object (Motorcycle) that violates a traffic signal } \\
\text { from the other side is turning left. }\end{array}$ & $1.92 \%$ \\
\hline 4 & Violating traffic signal & $\begin{array}{l}\text { Ego-vehicle is driving straight on the right of way at signalized } \\
\text { intersection. Target object (Motorcycle) that violates a traffic signal } \\
\text { from the other side is turning right. }\end{array}$ & $1.06 \%$ \\
\hline 5 & Lane-change & $\begin{array}{c}\text { Ego-vehicle is driving straight on the right of way at signalized } \\
\text { intersection. Target object (Motorcycle) is changing lanes into } \\
\text { ego-vehicle's lane in same direction. }\end{array}$ & $0.17 \%$ \\
\hline 6 & Stopping & $\begin{array}{l}\text { Ego-vehicle is driving straight on the right of way at signalized } \\
\text { intersection. Target object (Motorcycle) is stopping in front of } \\
\text { ego-vehicle in the same direction. }\end{array}$ & $0.14 \%$ \\
\hline
\end{tabular}


Table 14. Cont.

\begin{tabular}{|c|c|c|c|}
\hline No. & Provoking Events & Functional Scenarios & Ratios \\
\hline 7 & Violating traffic signal & $\begin{array}{c}\text { Ego-vehicle is turning left on the right of way at signalized } \\
\text { intersection. Target object (Motorcycle) that violates a traffic signal } \\
\text { from the left is driving straight. }\end{array}$ & $0.24 \%$ \\
\hline 8 & Violating traffic signal & $\begin{array}{l}\text { Ego-vehicle is turning left on the right of way at signalized } \\
\text { intersection. Target object (Motorcycle) that violates a traffic signal } \\
\text { from the right is driving straight. }\end{array}$ & $0.24 \%$ \\
\hline 9 & Violating traffic signal & $\begin{array}{l}\text { Ego-vehicle is turning left on the right of way at signalized } \\
\text { intersection. Target object (Motorcycle) that violates a traffic signal } \\
\text { from the other side is turning left. }\end{array}$ & $0.38 \%$ \\
\hline 10 & Violating traffic signal & $\begin{array}{l}\text { Ego-vehicle is turning left on the right of way at signalized } \\
\text { intersection. Target object (Motorcycle) that violates a traffic signal } \\
\text { from the other side is turning right. }\end{array}$ & $0.02 \%$ \\
\hline 11 & Lane-change & $\begin{array}{l}\text { Ego-vehicle is turning left on the right of way at signalized } \\
\text { intersection. Target object (Motorcycle) is changing lanes into } \\
\text { ego-vehicle's lane in the same direction. }\end{array}$ & $0.07 \%$ \\
\hline 12 & Stopping & $\begin{array}{l}\text { Ego-vehicle is turning left on the right of way at signalized } \\
\text { intersection. Target object (Motorcycle) is stopping in front of } \\
\text { ego-vehicle in the same direction. }\end{array}$ & $0.22 \%$ \\
\hline 13 & Violating traffic signal & $\begin{array}{l}\text { Ego-vehicle is turning right on the right of way at signalized } \\
\text { intersection. Target object (Motorcycle) that violates a traffic signal } \\
\text { from the other side is driving straight. }\end{array}$ & $0.17 \%$ \\
\hline 14 & Violating traffic signal & $\begin{array}{c}\text { Ego-vehicle is turning right on the right of way at signalized } \\
\text { intersection. Target object (Motorcycle) that violates a traffic signal } \\
\text { from the other side is turning left. }\end{array}$ & $0.10 \%$ \\
\hline 15 & Lane-change & $\begin{array}{l}\text { Ego-vehicle is turning right on the right of way at signalized } \\
\text { intersection. Target object (Motorcycle) is changing lanes into } \\
\text { ego-vehicle's lane in the same direction. }\end{array}$ & $0.05 \%$ \\
\hline 16 & Stopping & $\begin{array}{l}\text { Ego-vehicle is turning right on the right of way at signalized } \\
\text { intersection. Target object (Motorcycle) is stopping in front of } \\
\text { ego-vehicle in the same direction. }\end{array}$ & $0.10 \%$ \\
\hline
\end{tabular}

Regarding vehicle-to-bicycle functional scenarios, they accounted for $0.55 \%$ of the actual traffic accidents at intersection sections as shown in Table 15.

Table 15. Ratio of vehicle-to-bicycle functional scenarios from real accident data at intersection sections.

\begin{tabular}{cccc}
\hline No. & Provoking Events & Functional Scenarios & Ratios \\
\hline 1 & Jaywalking & $\begin{array}{c}\text { Ego-vehicle is driving straight on the right of way at signalized } \\
\text { intersection. Target object (Bicycle) is jaywalking on other side. }\end{array}$ & $0.22 \%$ \\
\hline 2 & Jaywalking & $\begin{array}{c}\text { Ego-vehicle is turning left on the right of way at signalized } \\
\text { intersection. Target object (Bicycle) is jaywalking on other side. }\end{array}$ & $0.12 \%$ \\
\hline 3 & Jaywalking & $\begin{array}{c}\text { Ego-vehicle is turning right on the right of way at signalized } \\
\text { intersection. Target object (Bicycle) is jaywalking on other side. }\end{array}$ & $0.22 \%$ \\
\hline
\end{tabular}

\section{Conclusions}

As the research and development activities of AVs have been active in recent years, developing test scenarios and methods has become necessary to evaluate and ensure the safety of AVs. Therefore, this study developed an automated vehicle test scenario derivation methodology using traffic accident data and an NLP technique. First, the level of AVs for the scenario to be developed was defined. The level of the automated vehicle was defined as level 4 of the SAE standards, which is high automation, and the ODD was defined as centered on urban arterial roads. Using the ODD defined above, the 
collected traffic accident data archived by the KNPA were used to extract traffic accidents in road sections and intersections of urban arterial roads. Additionally, the 'accident situation description' data described as text among the extracted traffic accident data were preprocessed. The main features were extracted from the preprocessed data using a feature extraction module based on TF-IDF vectorization. Furthermore, the main features of each object were extracted and classified according to the defined categories.

As a result, 16 functional test scenarios for urban arterials and 38 scenarios for intersections were generated on urban roads. The resulting test scenarios were validated by determining the number of traffic accident records that can be explained by the resulting test scenarios. That is, the resulting test scenarios are valid and represent a matching rate between the test scenarios and the increased number of traffic accident records. The resulting functional scenarios generated by the proposed methodology account for $43.69 \%$ and $27.63 \%$ of the actual traffic accidents for the urban arterial and intersection scenarios, respectively. Therefore, it is certain that the scenario-mining methodology proposed in this study can derive automated vehicle safety assessment scenarios from traffic accident data and it is inferred that it can be used to develop automated vehicle evaluation scenarios. This proposed methodology can fully utilize traffic accident data that include unsafe traffic conditions and is a systematic method for extracting edge cases, in which AVs need to be tested. Particularly, the methodology provides a practical method to analyze abundant text-based data written by police officers of traffic accident reports, which is barely possible because of the vastness of the data. Finally, this proposed methodology is universal for other traffic accident databases such as the German in-depth accident study (GIDAS), the initiative for the global harmonization of accident data (IGLAD), and the national automotive sampling system crashworthiness data system (NASS CDS), considering such datasets include the data elements used in this study.

\section{Recommendations for Future Research}

Although this study developed a methodology for mining functional scenarios for automated vehicle safety assessment using traffic accident data, NLP techniques, and a scenario for urban arterial roads, some limitations still exist. First, to derive various dangerous situations occurring in road sections, the scenario was derived using the accident situation described in the text of the traffic accident data of the KNPA. Although the traffic accident data represent the accident situation, there is a disadvantage in that detailed information, such as the speed of the vehicle at the time of the accident, the collision angle, and the location of the surrounding vehicles, remain unknown. If CCTV data or individual vehicle sensing data can be used in the future, more detailed scenarios can be derived and configured. Second, there is a limitation in that the methodology cannot be automated to select extracted features and type them by category. It is necessary to categorize the accident situation and derive characteristics using topic modeling or sentence-based embedding in the future. Third, there is a limitation in not evaluating and validating the developed functional scenario as an automated actual-vehicle experiment or simulation experiment. To solve this problem, it is necessary to evaluate and validate the appropriateness of the scenario through simulation or actual-vehicle tests by extending the developed functional scenario to logical and concrete scenarios. Lastly, the methodology needs to be advanced to address the cases in which multiple objects are involved at the same time, as this study focuses only on single object-related accident cases.

Author Contributions: Conceptualization, J.S., S.P. (Sangmin Park) and I.Y.; methodology, S.P. (Sangmin Park) and I.Y.; validation, H.J. and S.P. (Sungho Park); formal analysis, S.P. (Sangmin Park) and H.J.; investigation, H.J and S.P. (Sungho Park); writing—original draft preparation, S.P. (Sangmin Park) and H.J.; writing — review and editing, S.P. (Sangmin Park), J.S. and I.Y.; visualization, H.J.; supervision, J.S. and I.Y.; project administration, I.Y.; funding acquisition, I.Y. All authors have read and agreed to the published version of the manuscript.

Funding: This research study was supported by a grant (21PQOW-B152473-03) from the R\&D Program funded by the Ministry of Land, Infrastructure, and Transport of the Korean government. 
Institutional Review Board Statement: Not applicable.

Informed Consent Statement: Not applicable.

Data Availability Statement: The data used in this study cannot be made available due to the policy of the Korean National Police Agency (KNPA).

Conflicts of Interest: The authors declare no conflict of interest.

\section{Appendix A}

In the case of a vehicle as a target object, seven scenarios were developed in road sections (Table A1).

Table A1. Derived vehicle-to-vehicle functional scenarios in road sections.

\begin{tabular}{|c|c|c|c|c|}
\hline No. & Depictions & Maneuvers & Provoking Events & Functional Scenarios \\
\hline 1 & 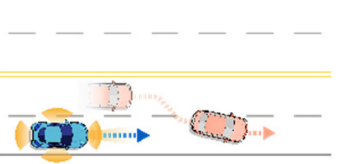 & Driving straight & Lane-change & $\begin{array}{l}\text { Ego-vehicle is driving straight at road } \\
\text { section. Target object (Vehicle) is } \\
\text { lane-changing into ego-vehicle's driving } \\
\text { lane ahead. }\end{array}$ \\
\hline
\end{tabular}

2
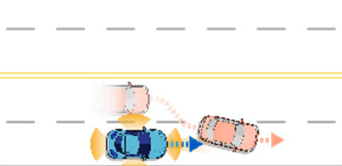

Driving straight

Abrupt lane-change

Ego-vehicle is driving straight at road section. Target object (Vehicle) is suddenly lane-changing into ego-vehicle's driving lane ahead.
3

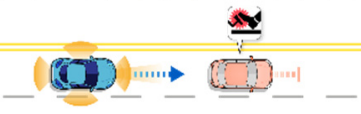

Driving straight

Stopping
Ego-vehicle is driving straight at road section. Target object (Vehicle) is stopping

in ego-vehicle's driving lane ahead.
4

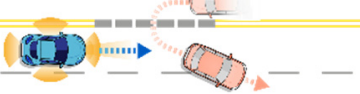

Driving straight

U-turn
Ego-vehicle is driving straight at road section. Target object (Vehicle) is making a

U-turn at ego-vehicle's driving lane ahead.

\begin{tabular}{|c|c|c|c|c|}
\hline 5 & 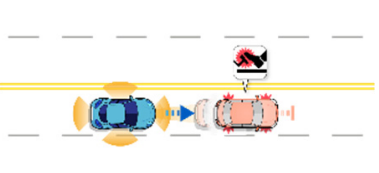 & Driving straight & Abrupt stopping & $\begin{array}{c}\text { Ego-vehicle is driving straight at road } \\
\text { section. Target object (Vehicle) is suddenly } \\
\text { stopping in ego-vehicle's driving lane } \\
\text { ahead. }\end{array}$ \\
\hline 6 & $\begin{array}{l}------1- \\
0010---1\end{array}$ & Driving straight & Drive over centerline & $\begin{array}{c}\text { Ego-vehicle is driving straight at road } \\
\text { section. Target object (Vehicle) is driving } \\
\text { over centerline into ego-vehicle's } \\
\text { driving lane. }\end{array}$ \\
\hline 7 & 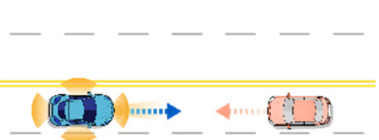 & Driving straight & Reversing & $\begin{array}{l}\text { Ego-vehicle is driving straight at road } \\
\text { section. Target object (Vehicle) is reversing } \\
\text { into ego-vehicle's driving lane. }\end{array}$ \\
\hline
\end{tabular}


In the case of a pedestrian as a target object, three scenarios were derived in road sections (Table A2).

Table A2. Derived vehicle-to-pedestrian functional scenarios in road sections.

\begin{tabular}{|c|c|c|c|c|}
\hline No. & Depictions & Maneuvers & Provoking Events & Functional Scenarios \\
\hline 1 & 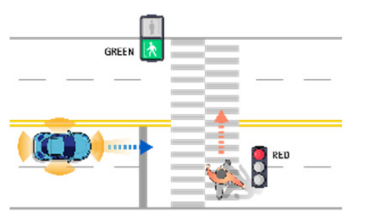 & Driving straight & Crossing & $\begin{array}{l}\text { Ego-vehicle is driving straight at road } \\
\text { section. Target object (Pedestrian) is } \\
\text { crossing ahead. }\end{array}$ \\
\hline 2 & $\begin{array}{l}------- \\
-\cos -\frac{-1}{6}-1\end{array}$ & Driving straight & Walking & $\begin{array}{l}\text { Ego-vehicle is driving straight at road } \\
\text { section. Target object (Pedestrian) is } \\
\text { walking in lane ahead. }\end{array}$ \\
\hline 3 & 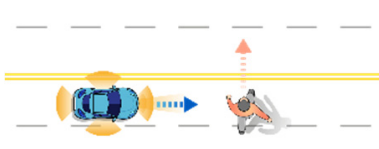 & Driving straight & Jaywalking & $\begin{array}{l}\text { Ego-vehicle is driving straight at road } \\
\text { section. Target object (Pedestrian) is } \\
\text { jaywalking ahead. }\end{array}$ \\
\hline
\end{tabular}

In the case of a motorcycle as a target object, three scenarios were derived in road sections (Table A3).

Table A3. Derived vehicle-to-motorcycle functional scenarios in road sections.

\begin{tabular}{|c|c|c|c|c|}
\hline No. & Depictions & Maneuvers & Provoking Events & Functional Scenarios \\
\hline 1 & $\begin{array}{l}------- \\
-(0)-\overline{1}-\overline{1}-1\end{array}$ & Driving straight & Lane-change & $\begin{array}{l}\text { Ego-vehicle is driving straight at road } \\
\text { section. Target object (Motorcycle) is } \\
\text { lane-changing into ego-vehicle's driving } \\
\text { lane. }\end{array}$ \\
\hline 2 & 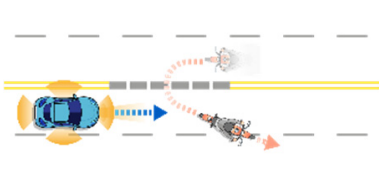 & Driving straight & U-turn & $\begin{array}{c}\text { Ego-vehicle is driving straight at road } \\
\text { section. Target object (Motorcycle) is } \\
\text { making a U-turn into ego-vehicle's } \\
\text { driving lane. }\end{array}$ \\
\hline 3 & 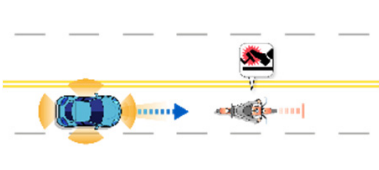 & Driving straight & Stopping & $\begin{array}{c}\text { Ego-vehicle is driving straight at road } \\
\text { section. Target object (Motorcycle) is } \\
\text { stopping in ego-vehicle's driving lane } \\
\text { ahead. }\end{array}$ \\
\hline
\end{tabular}

In the case of a bicycle as a target object, three scenarios were derived in road sections (Table A4). 
Table A4. Derived vehicle-to-bicycle functional scenarios in road sections.

\begin{tabular}{|c|c|c|c|c|}
\hline No. & Depictions & Maneuvers & Provoking Events & Functional Scenarios \\
\hline 1 & $\begin{array}{l}------- \\
-\frac{10}{10}-\frac{4}{4}-\end{array}$ & Driving straight & Crossing & $\begin{array}{c}\text { Ego-vehicle is driving straight at road } \\
\text { section. Target object (Bicycle) is crossing } \\
\text { ahead. }\end{array}$ \\
\hline 2 & $\begin{array}{l}------- \\
-(0)-----\end{array}$ & Driving straight & Driving straight & $\begin{array}{l}\text { Ego-vehicle is driving straight at road } \\
\text { section. Target object (Bicycle) is driving } \\
\text { straight in ego-vehicle's lane ahead. }\end{array}$ \\
\hline 3 & $\begin{array}{l}------- \\
-\cos ----6\end{array}$ & Driving straight & Reversing & $\begin{array}{l}\text { Ego-vehicle is driving straight at road } \\
\text { section. Target object (Bicycle) is reversing } \\
\text { into ego-vehicle's driving lane. }\end{array}$ \\
\hline
\end{tabular}

\section{Appendix B}

In the case of a vehicle as a target object, sixteen scenarios were developed in intersection sections (Table A5).

Table A5. Derived vehicle-to-vehicle functional scenarios in intersection sections.

\begin{tabular}{|c|c|c|c|c|}
\hline No. & Depictions & Maneuvers & Provoking Events & Functional Scenarios \\
\hline 1 & $\frac{1}{4}$ & Driving straight & Violating traffic signal & $\begin{array}{l}\text { Ego-vehicle is driving straight on the right } \\
\text { of way at signalized intersection. Target } \\
\text { object (Vehicle) that violates a traffic } \\
\text { signal from the left is driving straight. }\end{array}$ \\
\hline 2 & 1 & Driving straight & Violating traffic signal & $\begin{array}{l}\text { Ego-vehicle is driving straight on the right } \\
\text { of way at signalized intersection. Target } \\
\text { object (Vehicle) that violates a traffic } \\
\text { signal from the right is driving straight. }\end{array}$ \\
\hline 3 & $\int_{1}$ & Driving straight & Violating traffic signal & $\begin{array}{l}\text { Ego-vehicle is driving straight on the right } \\
\text { of way at signalized intersection. Target } \\
\text { object (Vehicle) that violates a traffic } \\
\text { signal from the other side is turning right. }\end{array}$ \\
\hline 4 & 1 & Driving straight & Violating traffic signal & $\begin{array}{l}\text { Ego-vehicle is driving straight on the right } \\
\text { of way at signalized intersection. Target } \\
\text { object (Vehicle) that violates a traffic } \\
\text { signal from the other side is turning left. }\end{array}$ \\
\hline
\end{tabular}


Table A5. Cont.

\begin{tabular}{llcl}
\hline No. Depictions & Maneuvers & Provoking Events & Functional Scenarios \\
\hline 5 & Driving straight & $\begin{array}{r}\text { Ego-vehicle is driving straight on the right } \\
\text { Lane-change }\end{array}$ & $\begin{array}{r}\text { of way at signalized intersection. Target } \\
\text { object (Vehicle) is changing lanes into } \\
\text { ego-vehicle's lane in the same direction. }\end{array}$
\end{tabular}

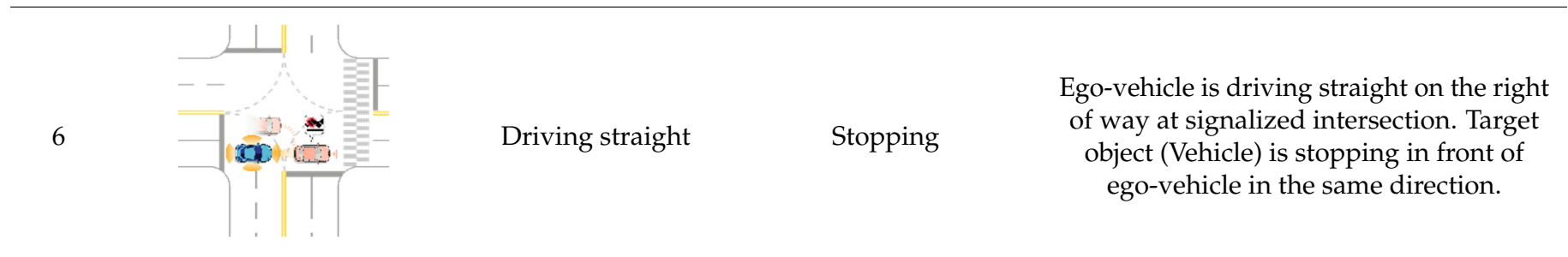

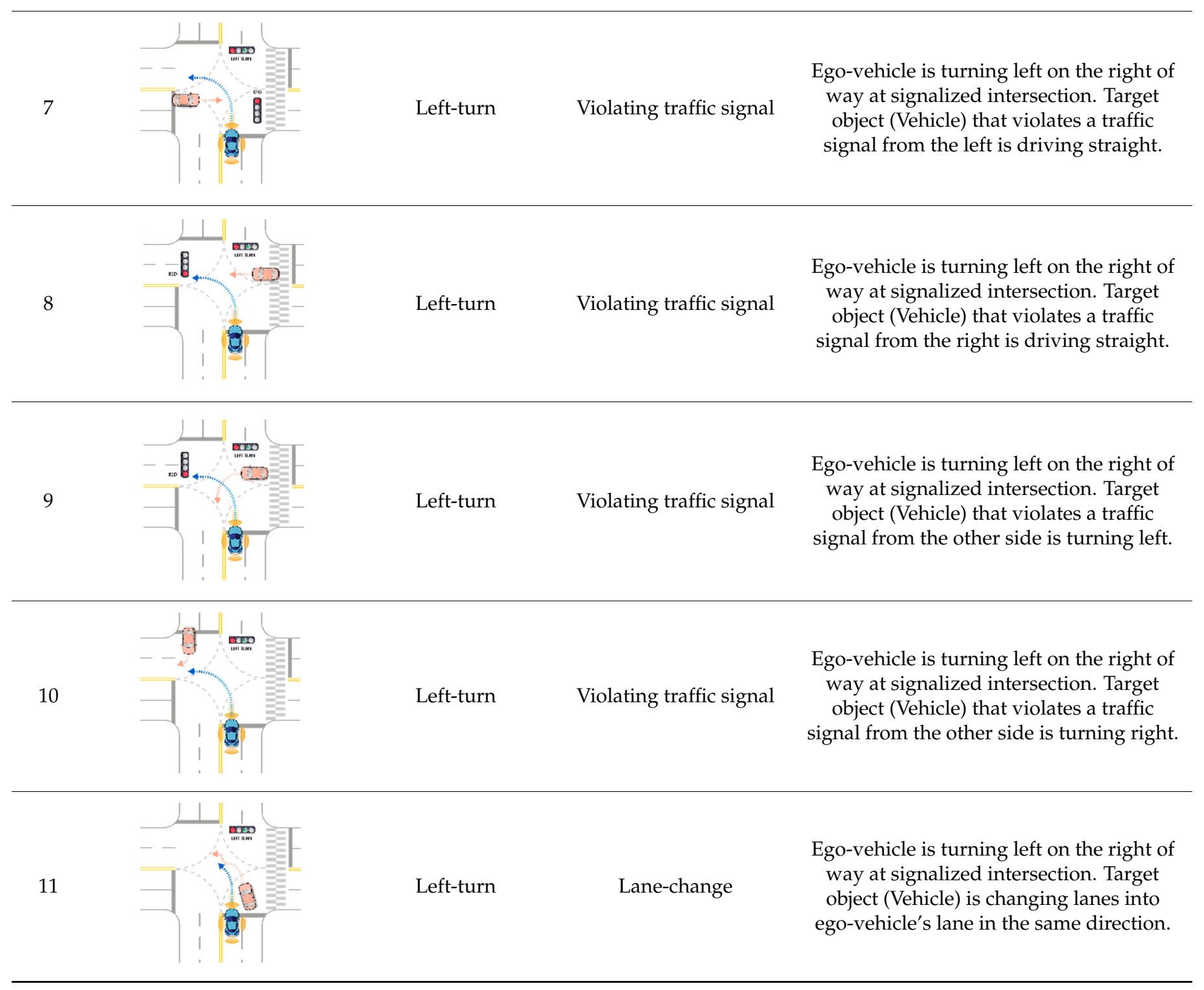


Table A5. Cont.

\begin{tabular}{llccc}
\hline No. Depictions & Maneuvers & Provoking Events & Functional Scenarios \\
\hline 12 & Left-turn & $\begin{array}{c}\text { Ego-vehicle is turning left on the right of } \\
\text { way at signalized intersection. Target } \\
\text { object (Vehicle) is stopping in front of } \\
\text { ego-vehicle in the same direction. }\end{array}$
\end{tabular}

13

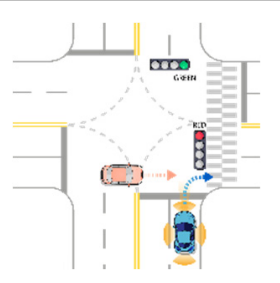

14

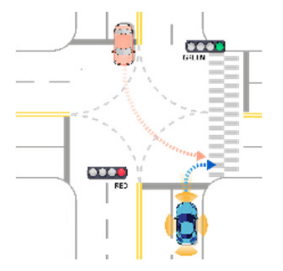

15

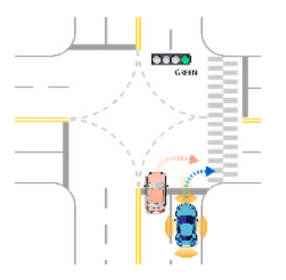

Right-turn

Right-turn

Violating traffic signal

Right-turn Violating traffic signal

Ego-vehicle is turning right on the right of way at signalized intersection. Target object (Vehicle) that violates a traffic signal from the other side is driving straight.

Ego-vehicle is turning right on the right of way at signalized intersection. Target object (Vehicle) that violates a traffic signal from the other side is turning left.
16

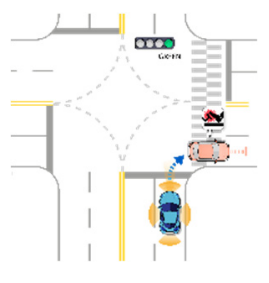

Ego-vehicle is turning right on the right of way at signalized intersection. Target object (Vehicle) is stopping in front of ego-vehicle in the same direction.

In the case of a pedestrian as a target object, three scenarios were developed in intersection sections (Table A6).

Table A6. Derived vehicle-to-pedestrian functional scenarios in intersections.

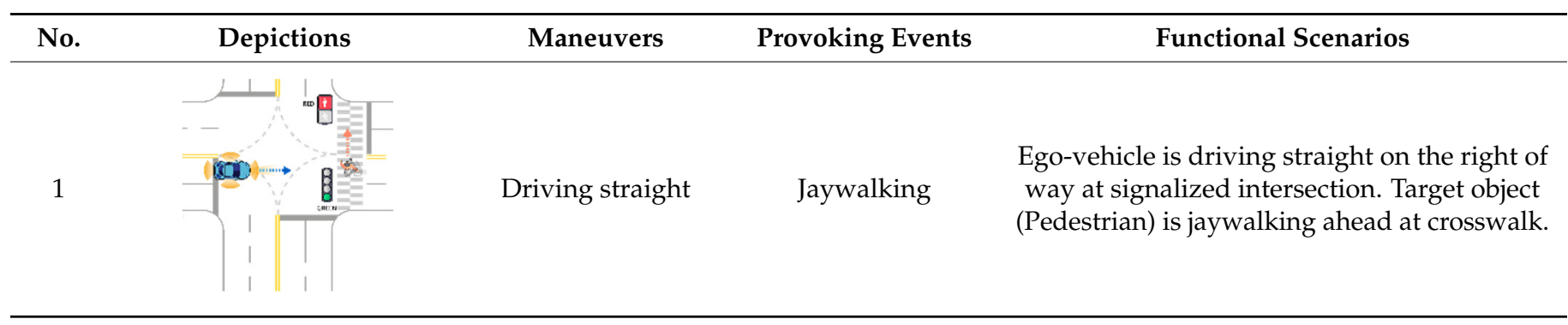


Table A6. Cont.

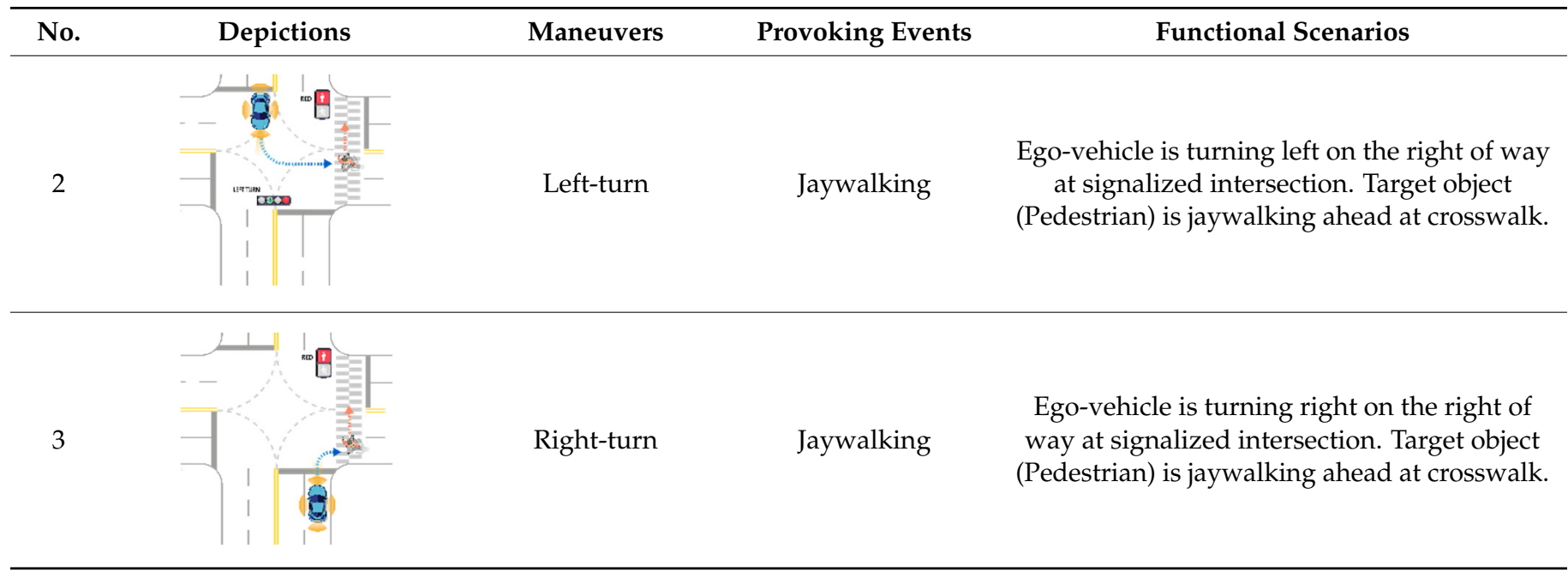

In the case of a motorcycle as a target object, sixteen scenarios were developed in intersection sections (Table A7).

Table A7. Derived vehicle-to-motorcycle functional scenarios in intersection sections.

\begin{tabular}{|c|c|c|c|c|}
\hline No. & Depictions & Maneuvers & Provoking Events & Functional Scenarios \\
\hline 1 & 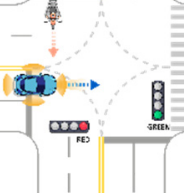 & Driving straight & Violating traffic signal & $\begin{array}{l}\text { Ego-vehicle is driving straight on the right } \\
\text { of way at signalized intersection. Target } \\
\text { object (Motorcycle) that violates a traffic } \\
\text { signal from the left is driving straight. }\end{array}$ \\
\hline 2 & 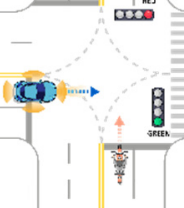 & Driving straight & Violating traffic signal & $\begin{array}{l}\text { Ego-vehicle is driving straight on the right } \\
\text { of way at signalized intersection. Target } \\
\text { object (Motorcycle) that violates a traffic } \\
\text { signal from the right is driving straight. }\end{array}$ \\
\hline
\end{tabular}

3

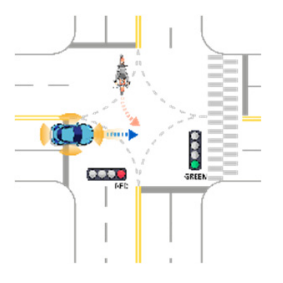

Driving straight

Violating traffic signal
Ego-vehicle is driving straight on the right of way at signalized intersection. Target object (Motorcycle) that violates a traffic signal from the other side is turning left.

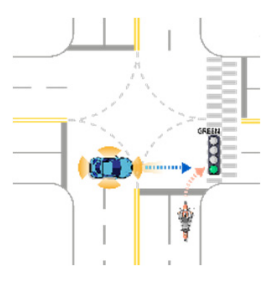

Ego-vehicle is driving straight on the right Driving straight Violating traffic signal of way at signalized intersection. Target object (Motorcycle) that violates a traffic signal from the other side is turning right. 
Table A7. Cont.

\begin{tabular}{llcl}
\hline No. Depictions & Maneuvers & Provoking Events & Functional Scenarios \\
Ego-vehicle is driving straight on the right \\
of way at signalized intersection. Target \\
object (Motorcycle) is changing lanes into \\
ego-vehicle's lane in the same direction.
\end{tabular}

\begin{tabular}{|c|c|c|c|c|}
\hline 6 & $x a,+\infty$ & Driving straight & Stopping & $\begin{array}{l}\text { Ego-vehicle is driving straight on the right } \\
\text { of way at signalized intersection. Target } \\
\text { object (Motorcycle) is stopping in front of }\end{array}$ \\
\hline & 1 & & & \\
\hline
\end{tabular}

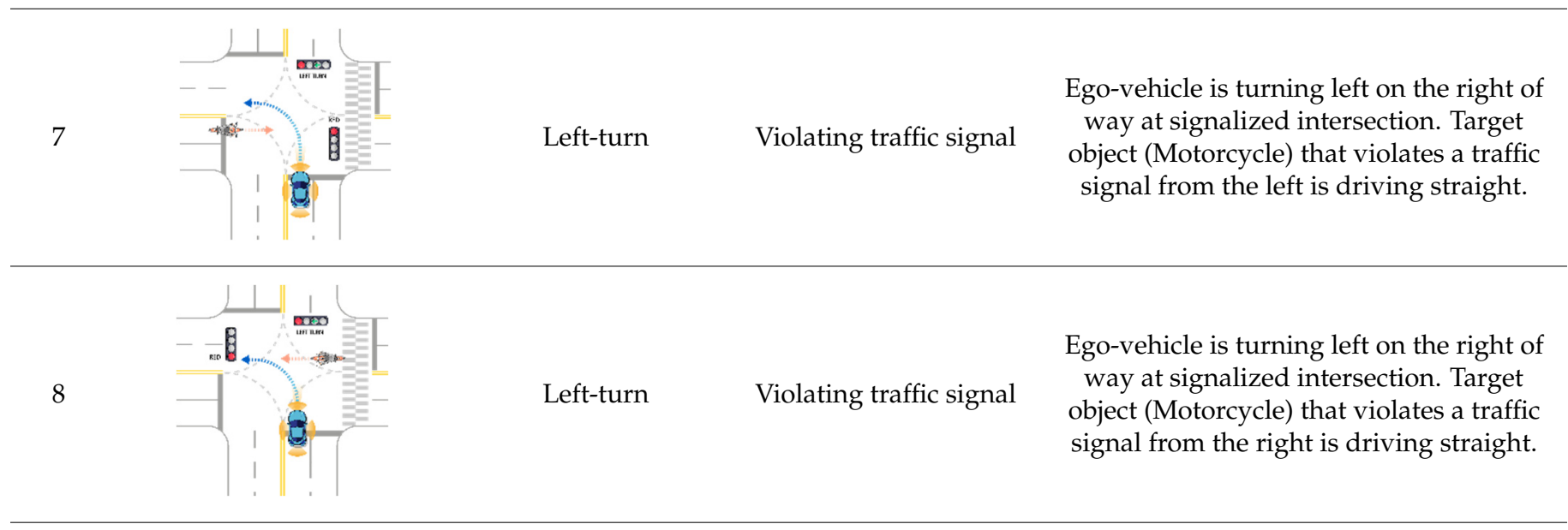

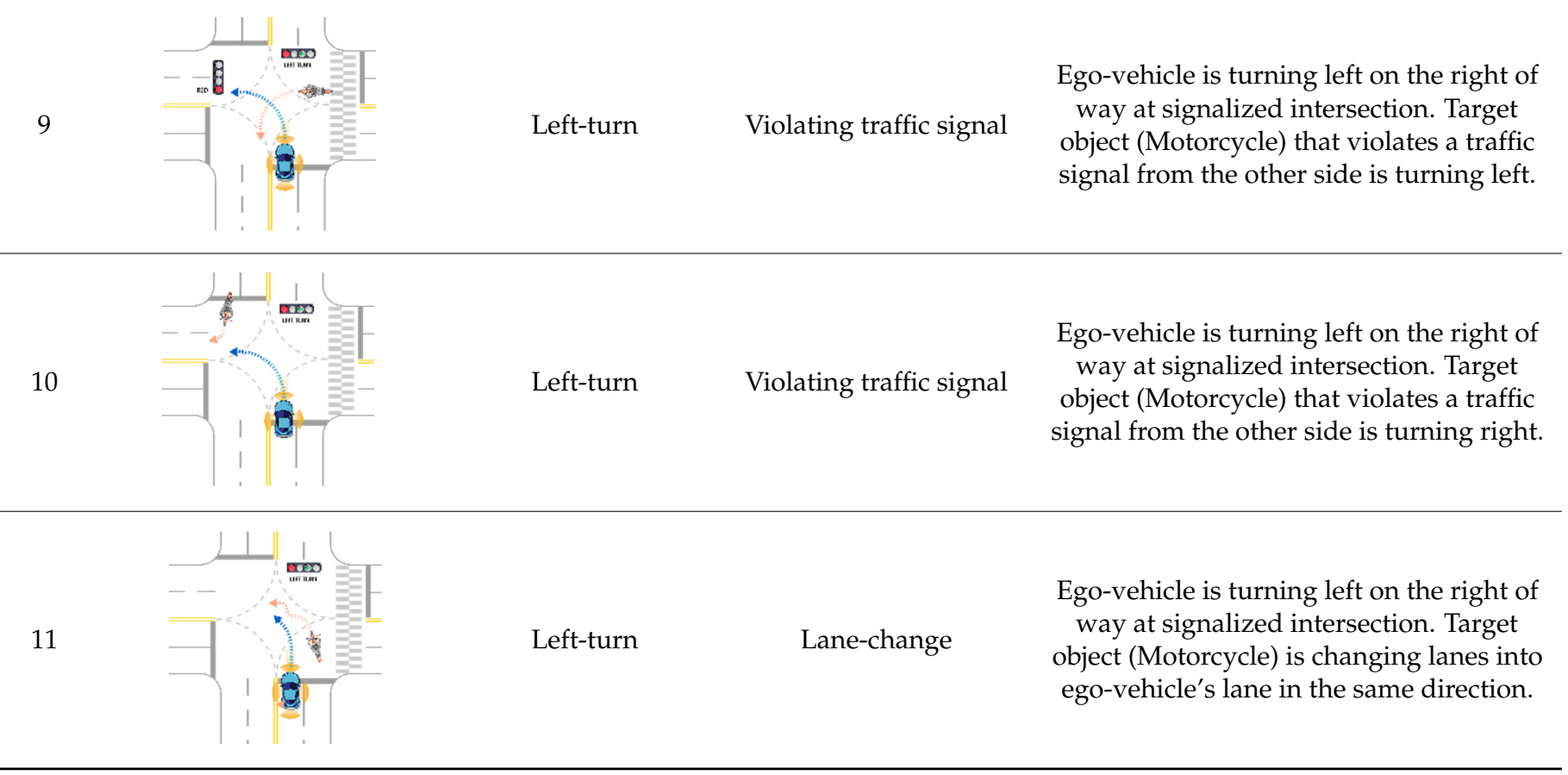


Table A7. Cont.

Maneuvers
Provoking Events $\begin{gathered}\text { Functional Scenarios } \\ \begin{array}{c}\text { Ego-vehicle is turning left on the right of } \\ \text { way at signalized intersection. Target object } \\ \text { (Motorcycle) is stopping in front of } \\ \text { ego-vehicle in the same direction. }\end{array}\end{gathered}$

15

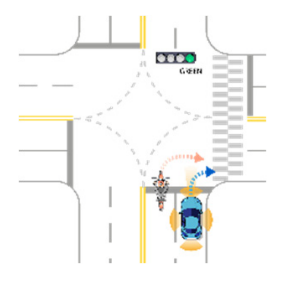

Ego-vehicle is turning right on the right of way at signalized intersection. Target object

(Motorcycle) is changing lanes into ego-vehicle's lane in the same direction.

In the case of a bicycle as a target object, three scenarios were developed in intersection sections (Table A8).

Table A8. Derived vehicle-to-bicycle functional scenarios in intersection sections.

\begin{tabular}{llccc}
\hline No. & Depictions & Maneuvers & Provoking Events & Functional Scenarios \\
\hline 1 & & Driving straight & $\begin{array}{c}\text { Jaywalking } \\
\text { Ego-vehicle is driving straight on the right } \\
\text { of way at signalized intersection. Target } \\
\text { object (Bicycle) is jaywalking on other side. }\end{array}$ \\
\hline
\end{tabular}


Table A8. Cont.

\begin{tabular}{llccc}
\hline No. & Depictions & Maneuvers & Provoking Events & Functional Scenarios \\
\hline 2 & Left-turn & $\begin{array}{c}\text { Ego-vehicle is turning left on the right of } \\
\text { Jaywalking }\end{array}$ & $\begin{array}{c}\text { (Bicycle) is jaywalking on other side. } \\
\text { (Bignalized intersection. Target object }\end{array}$ \\
\end{tabular}

3

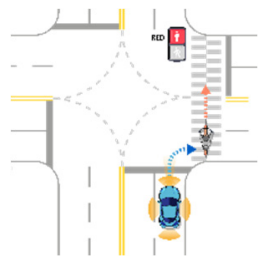

Right-turn Jaywalking
Ego-vehicle is turning right on the right of
way at signalized intersection. Target object

(Bicycle) is jaywalking on other side.

\section{References}

1. Thorn, E.; Kimmel, S.C.; Chaka, M.; Hamilton, B.A. A Framework for Automated Driving System Testable Cases and Scenarios; United States Department of Transportation, National Highway Traffic Safety Administration: Washington, DC, USA, 2018.

2. Chen, Z.; He, F.; Zhang, L.; Yin, Y. Optimal deployment of autonomous vehicle lanes with endogenous market penetration. Transp. Res. Part C Emerg. Technol. 2016, 72, 143-156. [CrossRef]

3. Chen, Z.; He, F.; Yin, Y.; Du, Y. Optimal design of autonomous vehicle zones in transportation networks. Transp. Res. Part B Methodol. 2017, 99, 44-61. [CrossRef]

4. Duarte, F.; Ratti, C. The Impact of Autonomous Vehicles on Cities: A Review. J. Urban Technol. 2018, 25, 3-18. [CrossRef]

5. Zhang, W.; Guhathakurta, S.; Fang, J.; Zhang, G. Exploring the impact of shared autonomous vehicles on urban parking demand: An agent-based simulation approach. Sustain. Cities Soc. 2015, 19, 34-45. [CrossRef]

6. Yamamoto, D.; Suganuma, N. Localization for autonomous driving on urban road. In Proceedings of the 2015 International Conference on Intelligent Informatics and Biomedical Sciences (ICIIBMS), Okinawa, Japan, 28-30 November 2015 ; pp. $452-453$.

7. Neurohr, C.; Westhofen, L.; Henning, T.; de Graaff, T.; Mohlmann, E.; Bode, E. Fundamental Considerations around ScenarioBased Testing for Automated Driving. In Proceedings of the 2020 IEEE Intelligent Vehicles Symposium (IV), Las Vegas, NV, USA, 19 October-13 November 2020; pp. 121-127.

8. Ma, W.; Qian, S. High-Resolution Traffic Sensing with Probe Autonomous Vehicles: A Data-Driven Approach. Sensors 2021, 21, 464. [CrossRef] [PubMed]

9. Saunier, N.; Sayed, T. Automated analysis of road safety with video data. Transp. Res. Rec. 2007, 2019, 57-64. [CrossRef]

10. Agrawal, R.; Nyamful, C. Challenges of Big Data Storage and Management. Glob. J. Inf. Technol. Emerg. Technol. 2016, 6, 1-10. [CrossRef]

11. Athanesious, J.; Srinivasan, V.; Vijayakumar, V.; Christobel, S.; Sethuraman, S.C. Detecting abnormal events in traffic video surveillance using superorientation optical flow feature. IET Image Process. 2020, 14, 1813-1821. [CrossRef]

12. Sohn, S.Y.; Shin, H. Pattern recognition for road traffic accident severity in Korea. Ergonomics 2001, 44, 107-117. [CrossRef]

13. Zhang, F.; Fleyeh, H.; Wang, X.; Lu, M. Construction site accident analysis using text mining and natural language processing techniques. Autom. Constr. 2019, 99, 238-248. [CrossRef]

14. Gyllenhammar, M.; Johansson, R.; Warg, F.; Chen, D.; Heyn, H.-M.; Sanfridson, M.; Söderberg, J.; Thorsén, A.; Ursing, S.; Ab, Z.; et al. Towards an Operational Design Domain That Supports the Safety Argumentation of an Automated Driving System. In Proceedings of the 10th European Congress on Embedded Real Time Systems (ERTS 2020), Toulouse, France, 29-31 January 2020; pp. 1-10.

15. Choi, Y.; Lim, J. Design for AEBS Test Scenario Applying Domestic Traffic Accidents. Int. J. Adv. Smart Converg. 2020, 9, 1-7.

16. Zhu, B.; Zhang, P.; Zhao, J.; Deng, W. Hazardous Scenario Enhanced Generation for Automated Vehicle Testing Based on Optimization Searching Method. IEEE Trans. Intell. Transp. Syst. 2021, 1-11. [CrossRef]

17. Nalic, D.; Eichberger, A.; Hanzl, G.; Fellendorf, M.; Rogic, B. Development of a Co-Simulation Framework for Systematic Generation of Scenarios for Testing and Validation of Automated Driving Systems. In Proceedings of the 2019 IEEE Intelligent Transportation Systems Conference (ITSC), Auckland, New Zealand, 27-30 October 2019; pp. 1895-1901.

18. Holland, J.C.; Sargolzaei, A. Verification of autonomous vehicles: Scenario generation based on real world accidents. In Proceedings of the 2020 SoutheastCon, Raleigh, NC, USA, 28-29 March 2020; p. 2.

19. So, J.J.; Park, I.; Wee, J.; Park, S.; Yun, I. Generating traffic safety test scenarios for automated vehicles using a big data technique. KSCE J. Civ. Eng. 2019, 23, 2702-2712. [CrossRef] 
20. Menzel, T.; Bagschik, G.; Maurer, A.M. Scenarios for Development, Test and Validation of Automated Vehicles. In Proceedings of the 2018 IEEE Intelligent Vehicles Symposium (IV), Changshu, China, 26-30 June 2018; pp. 1821-1827.

21. Waymo. Waymo Safety Report 2021. Available online: http:/ / waymo.com/safety/safety-report (accessed on 1 September 2021).

22. Chen, W.; Kloul, L. An ontology-based approach to generate the advanced driver assistance use cases of highway traffic. In Proceedings of the 10th International Joint Conference on Knowledge Discovery, Knowledge Engineering and Knowledge Management, Seville, Spain, 18-20 September 2018; Volume 2, pp. 75-83.

23. Ghodsi, Z.; Hari, S.K.S.; Frosio, I.; Tsai, T.; Troccoli, A.; Keckler, S.W.; Garg, S.; Anandkumar, A. Generating and Characterizing Scenarios for Safety Testing of Autonomous Vehicles. arXiv 2021, arXiv:2103.07403.

24. Riedmaier, S.; Ponn, T.; Ludwig, D.; Schick, B.; Diermeyer, F. Survey on Scenario-Based Safety Assessment of Automated Vehicles. IEEE Access 2020, 8, 87456-87477. [CrossRef]

25. Khurana, D.; Koli, A.; Khatter, K.; Singh, S. Natural Language Processing: State of The Art, Current Trends and Challenges. arXiv 2017, arXiv:1708.05148.

26. Kamerkar, N.; Patil, K.; Kale, A.; Patil, G.N. Text Mining Applied to Rail Accidents. Int. J. Future Revolut. Comput. Sci. Commun. Eng. 2018, 4, 383-386.

27. Cheng, M.Y.; Kusoemo, D.; Gosno, R.A. Text mining-based construction site accident classification using hybrid supervised machine learning. Autom. Constr. 2020, 118, 103265. [CrossRef]

28. Gao, L.; Wu, H. Verb-Based Text Mining of Road Crash Report. In Proceedings of the Transportation Research Board 92nd Annual Meeting, Washington, DC, USA, 13-17 January 2013.

29. Ali, F.; El-Sappagh, S.; Kwak, D. Fuzzy ontology and LSTM-based text mining: A transportation network monitoring system for assisting travel. Sensors 2019, 19, 234. [CrossRef]

30. Ali, F.; Ali, A.; Imran, M.; Naqvi, R.A.; Siddiqi, M.H.; Kwak, K.S. Traffic accident detection and condition analysis based on social networking data. Accid. Anal. Prev. 2021, 151, 105973. [CrossRef]

31. SAE International. Taxonomy and Definitions for Terms Related to Driving Automation Systems for On-Road Motor Vehicles; SAE International: Warrendale, PA, USA, 2018.

32. International Organization for Standardization. ISO/PAS 21448: 2019 Road Vehicles Safety of the Intended Functionality. Available online: https:/ / www.iso.org/standard/70939.html (accessed on 1 September 2021).

33. Pradha, S.; Halgamuge, M.N.; Tran Quoc Vinh, N. Effective text data preprocessing technique for sentiment analysis in social media data. In Proceedings of the 2019 11th International Conference on Knowledge and Systems Engineering (KSE), Da Nang, Vietnam, 24-26 October 2019.

34. Shah, F.P.; Patel, V. A review on feature selection and feature extraction for text classification. In Proceedings of the 2016 International Conference on Wireless Communications, Signal Processing and Networking (WiSPNET), Chennai, India, 23-25 March 2016; pp. 2264-2268.

35. Zhang, W.; Yoshida, T.; Tang, X. A comparative study of TF*IDF, LSI and multi-words for text classification. Expert Syst. Appl. 2011, 38, 2758-2765. [CrossRef]

36. Federal Ministry for Economic Affairs and Energy (BMWi). PEGASUS RESEARCH PROJECT. Available online: https://www. pegasusprojekt.de/en/about-PEGASUS (accessed on 19 August 2021).

37. Federal Ministry for Economic Affairs and Energy (BMWi). PEGASUS Symposium 2019. PEGASUS METHOD. Available online: https:/ / www.pegasusprojekt.de/files/tmpl/Pegasus-Abschlussveranstaltung/PEGASUS-Gesamtmethode.pdf (accessed on 19 August 2021).

38. Menzel, T.; Bagschik, G.; Isensee, L.; Schomburg, A.; Maurer, M. From functional to logical scenarios: Detailing a keyword-based scenario description for execution in a simulation environment. In Proceedings of the 2019 IEEE Intelligent Vehicles Symposium (IV), Paris, France, 9-12 June 2019; pp. 2383-2390. 